\title{
Article
}

\section{The mTOR inhibitor AZD8055 overcomes tamoxifen resistance in breast cancer cells by down-regulating HSPB8}

\author{
Jia-jie SHI ${ }^{1,2}$, Si-meng $\mathrm{CHEN}^{1}$, Chen-liang GUO ${ }^{1}$, Yi-xue $\mathrm{LI}^{3}$, Jian $\mathrm{DING}^{1,2, *}$, Ling-hua MENG ${ }^{1,2, *}$ \\ ${ }^{1}$ Division of Anti-tumor Pharmacology, State Key Laboratory of Drug Research, Shanghai Institute of Materia Medica, Chinese Academy \\ of Sciences, Shanghai 201203, China; ${ }^{2}$ University of Chinese Academy of Sciences, Beijing 100049, China; ${ }^{3}$ Shanghai Center for \\ Bioinformation Technology, Shanghai 201203, China
}

\begin{abstract}
Tamoxifen, an important endocrine therapeutic agent, is widely used for the treatment of estrogen receptor positive (ER $\left.{ }^{+}\right)$breast cancer. However, de novo or acquired resistance prevents patients from benefitting from endocrine approaches and necessitates alternative treatments. In this study, we report that small heat protein beta-8 (HSPB8) may serve as an important molecule in tamoxifen resistance. HSPB8 expression is enhanced in MCF-7 cells resistant to tamoxifen (MCF-7/R) compared to parent cells. Moreover, high expression of HSPB8 associates with poor prognosis in $\mathrm{ER}^{+}$breast cancer patients but not in patients without classification. Stimulating ER signaling by heterogeneous expression of ERa or 17ß-estradiol promotes HSPB8 expression and reduces the cell population in $\mathrm{G}_{1}$ phase. In contrast, blockage of ER signaling by tamoxifen down-regulates the expression of HSPB8. In addition, knocking down HSPB8 by specific siRNAs induces significant cell cycle arrest at $\mathrm{G}_{1}$ phase. AZD8055 was found to be more potent against the proliferation of MCF-7/R cells than that of parent cells, which was associated with down-regulation of HSPB8. We found that the anti-proliferative activity of AZD8055 was positively correlated with the HSPB8 expression level in ER ${ }^{+}$breast cancer cells. Thus, AZD8055 was able to overcome tamoxifen resistance in breast cancer cells, and the expression of HSPB8 may predict the efficacy of AZD8055 in $\mathrm{ER}^{+}$breast cancer. This hypothesis deserves further investigation.
\end{abstract}

Keywords: tamoxifen resistance; HSPB8; AZD8055; estrogen receptor; mTOR inhibitor

Acta Pharmacologica Sinica (2018) 39: 1338-1346; doi: 10.1038/aps.2017.181; published online 18 Jan 2018

\section{Introduction}

Breast cancer is the most prevalent malignant tumor and the second leading cause of mortality in female cancer patients ${ }^{[1]}$. Approximately $80 \%$ of breast cancers are estrogen receptor positive $\left(\mathrm{ER}^{+}\right)$, and tamoxifen was approved 40 years ago as a promising therapy for this type of breast cancer ${ }^{[2,3]}$. Tamoxifen reduces the risk of death by one-third, and more than $50 \%$ of $\mathrm{ER}^{+}$patients are believed to be benefited from the therapy ${ }^{[4]}$. Today, tamoxifen is widely used as a standard therapy for $\mathrm{ER}^{+}$ breast cancer in premenopausal women.

Although endocrine therapy benefits a large portion of $\mathrm{ER}^{+}$ patients, the effects are diminished with the development of primary or secondary resistance ${ }^{[5]}$. Approximately $50 \%$ of $\mathrm{ER}^{+}$ breast cancer patients who positively respond to endocrine

\footnotetext{
*To whom correspondence should be addressed.

E-mail Ihmeng@simm.ac.cn (Ling-hua MENG); jding@simm.ac.cn (Jian DING)

Received 2017-09-09 Accepted 2017-10-31
}

interventions at the beginning will eventually become resistant. Tamoxifen, as the first drug targeting ER for the treatment of $\mathrm{ER}^{+}$breast cancers, also suffers from the challenge of development of resistance ${ }^{[6]}$. Alterations in co-regulatory proteins or kinase signaling transduction are common in resistant cells ${ }^{[7,8]}$. A high-throughput cell-based screening revealed that the small heat shock protein HSPB8 played an important role in the development of resistance to tamoxifen ${ }^{[9]}$. HSPB8 executes its chaperone activity under heat stress, preventing the aggregation or accumulation of denatured proteins ${ }^{[10,11]}$. HSPB8 has also been reported to participate in the quality control of proteins in a non-canonical manner, where the HSPB8Bag3 complex induces eIF2a phosphorylation and stimulates autophagy ${ }^{[12]}$. Gonzalez-Malerva et $a l^{[9]}$ demonstrated that HSPB8 protected MCF-7 cells from tamoxifen treatment and promoted cell proliferation by blocking autophagy. Further studies reveal that silencing HSBP8 induced autophagy ${ }^{[9,13]}$. The exact mechanism of HSPB8 in tamoxifen resistance remains elusive. 
The mammalian target of rapamycin (mTOR) is a serine/ threonine protein kinase, which plays a critical role in the signaling pathways associated with cell survival, proliferation, metabolism, angiogenesis and growth ${ }^{[14,15]}$. The mTOR pathway is abnormally activated in many tumor types due to alterations in key factors both upstream and downstream of mTOR. Inhibition of mTOR has been shown to be a promising strategy for the treatment of breast cancer, and the first generation mTOR inhibitor rapalogs have been approved for treatment of advanced hormone receptor-positive and HER2negative breast cancer in combination with exemestane, as well as for advanced renal cell carcinoma. However, the therapeutic efficacy of the rapalogs is limited as mono-therapy, which is possibly due to the incomplete inhibition of $\mathrm{mTOR}^{[16]}$. The second-generation mTOR inhibitors inhibit the kinase activity of both mTORC1 and mTORC2 and are considered to possess more potent anticancer activity than the rapalogs. AZD8055 emerged as a first-in-class, orally available, specific mTOR kinase inhibitor with powerful antitumor activity in vitro and in vivo ${ }^{[17]}$. Jordan et al demonstrated that AZD8055 might be able to treat breast cancers resistant to endocrine therapy agents, including tamoxifen and fulvestrant ${ }^{[18]}$. Moreover, inhibition of mTOR with AZD8055 or RAD001 resulted in more potent activity in tamoxifen-resistant cells than in parent cells ${ }^{[18]}$, but the mechanisms remain unclear.

To investigate the mechanism of resistance to tamoxifen and identify potential strategies to overcome the resistance, we established tamoxifen-resistant MCF-7 (MCF-7/R) cells and found that HSPB8 is overexpressed in MCF-7/R cells. We further determined that the HSPB8 expression level is associated with poor prognosis in $\mathrm{ER}^{+}$breast cancer patients. The activity of mTOR inhibitors was positively correlated with HSPB8 in NCI60 screening. Accordingly, AZD8055 was more potent against the proliferation of MCF-7/R cells than that of parent cells, which was accompanied with down-regulation of HSPB8. We also found that the anti-proliferative activity of AZD8055 was positively correlated with the expression level of HSPB8 in a panel of $\mathrm{ER}^{+}$breast cancer cells.

\section{Materials and methods}

\section{Compounds and reagents}

AZD8055 was purchased from Selleck Chemicals (Houston, TX, USA). (Z)-4-Hydroxytamoxifen was obtained from Sigma (St Louis, MO, USA). All these compounds were dissolved in DMSO as stock solutions and stored at $-20^{\circ} \mathrm{C}$.

Antibodies against HSPB8, p70S6K, phospho-p70S6K (T389), $\mathrm{Rb}$, phospho-Rb (S807/11), and Cyclin D1 were purchased from Cell Signaling Technology (Danvers, MA, USA). Anti$\beta$-actin antibody was purchased from Sigma (St Louis, MO, USA). Anti-estrogen receptor alpha antibody was obtained from Abcam (Canary Wharf, London, UK).

\section{Cell culture}

The human cell lines T47D, ZR-75-30, ZR-75-1, MDA-MB-453, MDA-MB-231, BT-474, HCC38, MDA-MB-361, SK-BR-3 and MDA-MB-468 were obtained from the American Type Culture
Collection (ATCC), and JIMT-1, EFM-192A, CAL-51, EFM19, S1 and MFM223 were obtained from the Leibniz Institute - German Collection of Microorganisms and Cell Cultures (DSMZ). All the cell lines were cultured according to the suppliers' instructions. MCF-7 cells were obtained from ATCC and cultured in DMEM medium containing 10\% FBS. MCF-7 cells resistant to tamoxifen (MCF-7/R) were established by incubation with stepwise-increasing concentrations of tamoxifen. MCF-7/R cells were cultured in phenol-red free DMEM supplemented with $10 \%$ charcoal-stripped fetal calf serum and $10^{-5} \mathrm{~mol} / \mathrm{L}$ 4-hydroxytamoxifen.

\section{Cell proliferation assays}

Cell proliferation was evaluated using the sulforhodamine B (SRB, Sigma, ST, USA) assay as described previously ${ }^{[19]}$. Briefly, cells were seeded in 96-well plates at an appropriate density. Diluted compounds were added to the cells on the next day. After incubation at $37^{\circ} \mathrm{C}$ for $72 \mathrm{~h}$, cells were fixed with $10 \%$ pre-cooled trichloroacetic acid. Then, the cells were washed with distilled water and stained with SRB at $4 \mathrm{mg} / \mathrm{mL}$. SRB was dissolved in $10 \mathrm{mmol} / \mathrm{L}$ Tris, and $O D$ values were measured at $560 \mathrm{~nm}$. The inhibitory rate was calculated using the formula: $\left(O D_{\text {control cells }}-O D_{\text {treated cells }}\right) / O D_{\text {con- }}$ trol cells $\times 100 \%$ or $\left(O D_{\text {control cells }}-O D_{\text {treated cells }}\right) /\left(O D_{\text {control cells }}-O D_{\text {Day0 }}\right.$ cells $) \times 100 \%$. IC $\mathrm{IC}_{50}$ or $\mathrm{GI}_{50}$ values were calculated by four-parameter concentration-response curve fitting.

\section{Correlation analysis}

The protein levels of HSPB8 were detected by Western blotting, and the relative intensity was quantified by normalization to the intensity of $\beta$-actin in each cell line. The anti-proliferative activity of AZD8055 was represented by $\mathrm{GI}_{50}$ values, which were determined using the SRB assay. The relative protein intensity values and $\mathrm{GI}_{50}$ values were analyzed using the following formula: relative value $=\left(\right.$ value $\left._{\text {individual }}{ }^{- \text {value }_{\text {mean }}}\right)$ / value $_{\text {mean. }}$ Pearson correlation analyses were also conducted.

\section{Cell transfection}

\section{siRNA transfection}

Cells were seeded in 6-well plates. On the next day, the medium was replaced with fresh medium, and siRNA transfections were performed using Lipofectamine RNAi max (Invitrogen) following the manufacturer's instructions. The target sequences of HSPB8 were as follows: siHSPB8\#1, 5'-GGAGGUGUCUGGCAAACAUTT-3' and siHSPB8\#2, 5'-GGGUCUGCUGAUCAUCGAATT-3'.

\section{Plasmids transfection}

Plasmids plx301 (\#25895), pDONR223-HSPB8 (\#23606), and pcDNA-HA-ER WT (\#49498) were obtained from Addgene. pcDNA3.1 (+) (V790-20) was purchased from Invitrogen. HSPB8 was cloned into the plx301 vector from pDONR223HSPB8 via LR Clonase (Invitrogen, CA, USA). Cells seeded in 6-well plates were received fresh medium on the next day and were transfected with the indicated plasmids using Lipofectamine 2000 (Invitrogen, CA, USA) according to the manu- 
facturer's instructions.

\section{Western blotting}

Cell lysates were prepared with lysis buffer $(50 \mathrm{mmol} / \mathrm{L}$ Tris ( $\mathrm{pH}$ 7.4), $150 \mathrm{mmol} / \mathrm{L} \mathrm{NaCl}, 1 \% \mathrm{NP}-40,0.25 \%$ sodium deoxycholate) and then centrifuged at $20000 \times g$ for $15 \mathrm{~min}$ at $4{ }^{\circ} \mathrm{C}$. The supernatant was collected for heating at $100{ }^{\circ} \mathrm{C}$ for $10 \mathrm{~min}$. Standard Western blotting was performed to detect the indicated proteins.

\section{Cell cycle analysis}

For gene-transfected cells, cells were seeded at a density of $2 \times 10^{5} /$ well in 6-well plates and incubated for $24 \mathrm{~h}$. Then, the designated genes were transfected, and cells were further incubated for $48 \mathrm{~h}$. For compound-treated cells, $2 \times 10^{5}$ cells were seeded in 6-well plates and treated with test compounds on the next day for the indicated time. The cells were harvested and fixed in $70 \%$ ethanol overnight at $-20{ }^{\circ} \mathrm{C}$ and stained with $5 \mu \mathrm{g} / \mathrm{mL}$ propidium iodide (PI) for $30 \mathrm{~min}$. Cell cycle distributions were evaluated using a Becton-Dickinson FACS Calibur flow cytometer (BD Biosciences, CA, USA), and the raw data were analyzed using Flowjo software.

\section{Statistical analysis}

Quantitative data are shown as the mean $\pm S D$, with significance determined by Student's $t$-test. $P$ values of $<0.05$ were considered statically significant. All statistical analyses were performed using GraphPad Prism software (California, CA, USA).

\section{Results}

Overexpression of HSPB8 contributes to the proliferation of MCF-7 cells resistant to tamoxifen

To understand the role of HSPB8 and explore strategies to overcome tamoxifen resistance, we established a tamoxifenresistant MCF-7 cell line named MCF-7/R. The MCF-7/R cells were more tolerant to tamoxifen than parent cells, and the $\mathrm{IC}_{50}$ of the MCF-7/R cells was approximately two fold that of the parent cells (Figure 1A). Consistent with a previous report ${ }^{[9]}$, the HSPB8 protein level in MCF-7/R cells was higher than that in MCF-7 cells (Figure 1B). To investigate the role of HSPB8 in the proliferation of MCF-7/R cells, we knocked down HSPB8 with specific siRNAs. As shown in Figure $1 \mathrm{C}$ and Figure 1D, down-regulation of HSPB8 resulted in an accumulation of cells in $\mathrm{G}_{1}$ phase, which was accompanied with decreased phosphorylation of Rb. Thus, HSPB8 is overexpressed in MCF-7/R cells, and down-regulation of HSPB8 induces cell cycle arrest at $G_{1}$ phase.

HSPB8 overexpresses and correlates with poor prognosis in $\mathrm{ER}^{+}$ patients

As MCF-7/R cells overexpress HSPB8, revealing their func-
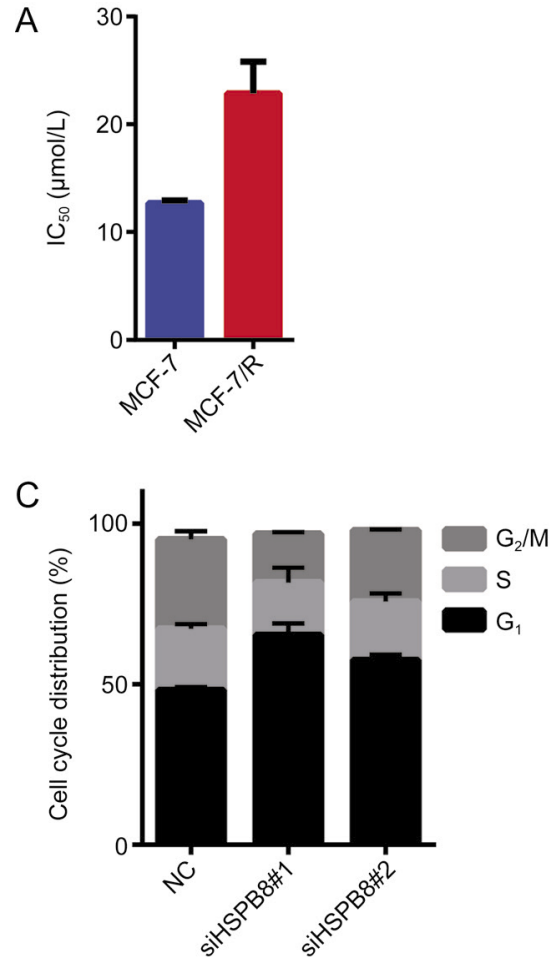

B
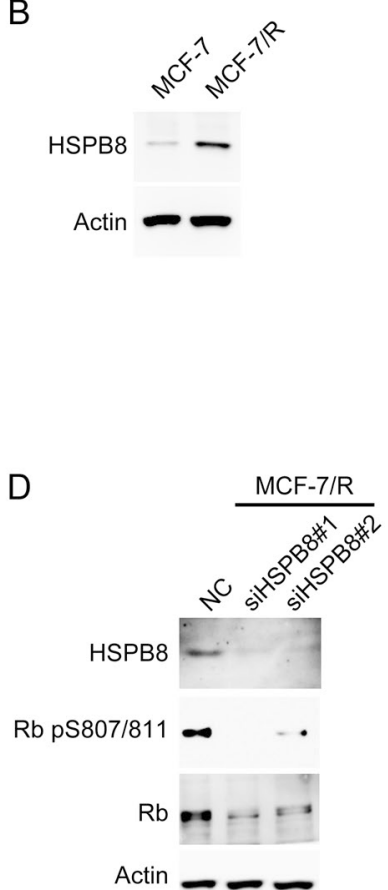

Figure 1. Overexpression of HSPB8 contributes to the proliferation of MCF-7 cells resistant to tamoxifen. (A) An MCF-7 cell line resistant to tamoxifen (MCF-7/R) was established in a stepwise-increasing concentration manner. MCF-7/R cells were treated with tamoxifen for $72 \mathrm{~h}$, and cell viability was assessed by the SRB assay. (B) Cell lysates from MCF-7 and MCF-7/R were prepared, and HSPB8 was detected by Western blotting. (C, D) MCF-7/R cells were transfected with siRNAs targeting HSPB8 for $48 \mathrm{~h}$. Cell cycle distribution was analyzed by flow cytometry (C), and the indicated proteins were detected by Western blotting (D). 

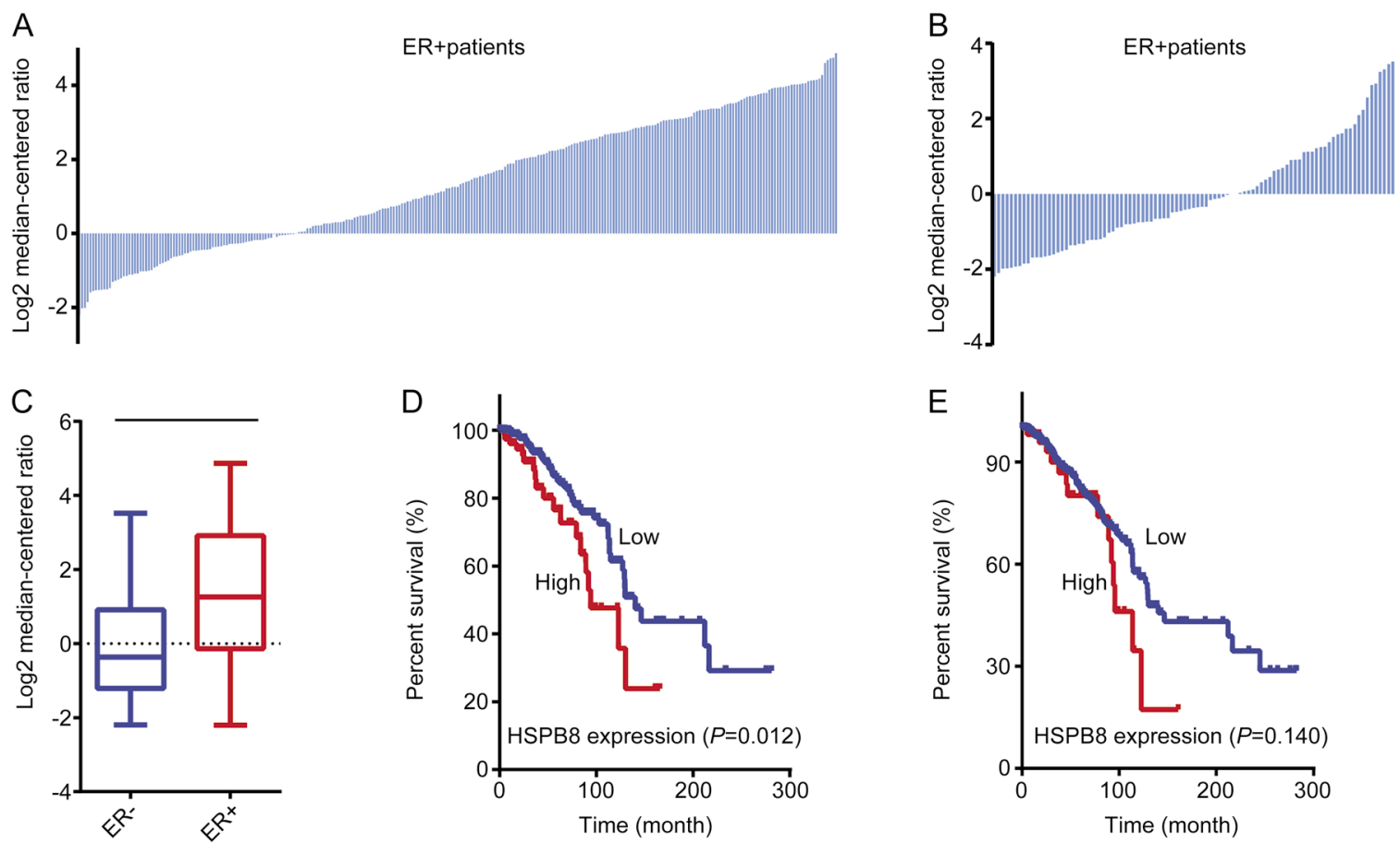

Figure 2. HSPB8 overexpresses and correlates with poor prognosis in $\mathrm{ER}^{+}$patients. HSPB8 expression levels in $E R^{+}$patients $(A)$ and in $E R^{-}$patients (B) were retrieved from the Oncomine database. (C) Mean HSPB8 expression level in $E R^{+}$and ER patients. ${ }^{* * *} P<0.0001$. The overall survival curves of patients with different HSPB8 expression were plotted using the cBioPortal for Cancer Genomics database in the group of $\mathrm{ER}^{+}$patients (D) and in patients without classification (E).

tion in regulating cell cycle progression, we retrieved the HSPB8 expression profile in breast cancer patients from the Oncomine database (https://www.oncomine.org/resource/ login.html). As shown in Figure 2A, in a cohort of $273 \mathrm{ER}^{+}$ patients, 194 patients displayed enhanced HSPB8 expression. However, the numbers of patients expressing high or low levels of HSPB8 were similar (39 vs 56) in ER ${ }^{-}$patients (Figure 2B). Moreover, the HSPB8 expression level in $\mathrm{ER}^{+}$ patients tends to be much higher than that in ER patients (Figure $2 \mathrm{C}$ ). These results indicate that HSPB8 is overexpressed in $\mathrm{ER}^{+}$patients.

To further explore the potential influence of HSPB8 expression on the survival of breast cancer patients, we conducted a Kaplan-Meier analysis. Data for HSPB8 expression and overall survival of breast cancer patients were obtained from the cBioPortal for Cancer Genomics database (http://www. cbioportal.org/). The patients were divided into two groups according to HSPB8 expression level in tumor tissues using a cut-off of a 1.2-fold z-score. In a cohort of $591 \mathrm{ER}^{+}$patients, 78 patients with high HSPB8 expression were revealed to have worse prognosis, with a median survival time of 94 months compared to 140 months in patients with low HSPB8 expression (Figure 2D). However, the difference in median survival time between patients with high and low HSPB8 expression level was not statistically significant in a cohort containing $1089 \mathrm{ER}^{+}$and $\mathrm{ER}^{-}$patients (96 months and 130 months respectively, Figure 2E). Therefore, HSPB8 up-regulation was correlated with poor survival in $\mathrm{ER}^{+}$patients.

\section{ER promotes HSPB8 expression}

Since we found that HSPB8 is overexpressed in most $\mathrm{ER}^{+}$ patients, we would like to explore the effect of ER signaling on HSPB8. We treated MCF-7 cells with 17 $\beta$-estradiol, a ligand of ER, for $48 \mathrm{~h}$ and then detected the HSPB8 protein level and analyzed the cell population in $G_{1}$ phase. As shown in Figure 3A, 17 $\beta$-estradiol enhanced HSPB8 expression and reduced cell populations in $\mathrm{G}_{1}$ phase (Figure $3 \mathrm{~B}$ ). In addition, we constructed MCF-7 cells overexpressing ERa (Figure 3C) and found that ERa expression resulted in enhanced HSPB8 level and reduced cell populations in $G_{1}$ phase (Figure $3 C$, $3 \mathrm{D})$, which is similar to the observations upon $17 \beta$-estradiol treatment in MCF-7 cells. In contrast, tamoxifen $(1 \mu \mathrm{mol} / \mathrm{L})$ significantly reduced the expression of HSPB8 in MCF-7 cells and induced $G_{1}$ phase arrest (Figure 3E, 3F). Collectively, we demonstrated that ER could promote HSPB8 expression and cell cycle progression.

\section{Knocking down of HSPB8 induces cell cycle arrest}

To define the role of HSPB8 in $\mathrm{ER}^{+}$cells, HPSB8 expression was down-regulated by specific siRNAs in MCF-7 and T47D cells (Figure 4B). Decreased HSPB8 expression induced the accumulation of cells in the $G_{1}$ phase in both cell lines (Figure $4 \mathrm{~A})$. The cell population in the $\mathrm{G}_{1}$ phase increased from $49 \%$ to $88 \%$ (siHSPB8\#1) and 67\% (siHSPB8\#2) in MCF-7 cells. A similar phenomenon was observed in T47D cells (Figure 4A, right panel). Accordingly, down-regulation of HSPB8 led to a decrease in the expression of cyclin D1 and phosphorylated 
A

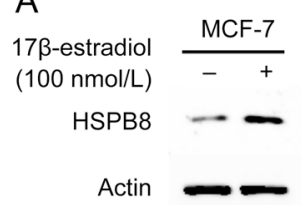

D

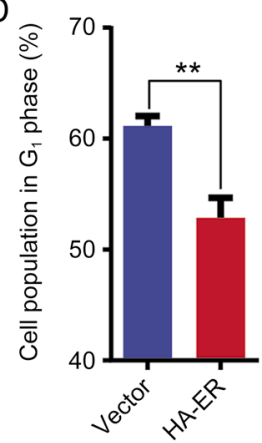

$\mathrm{B}$

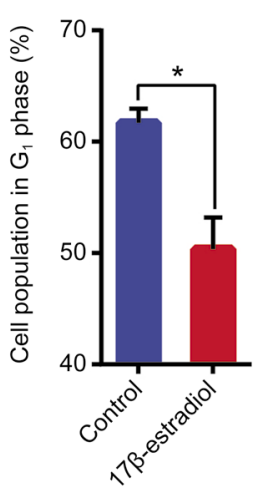

$\mathrm{E}$

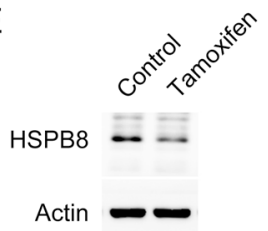

C
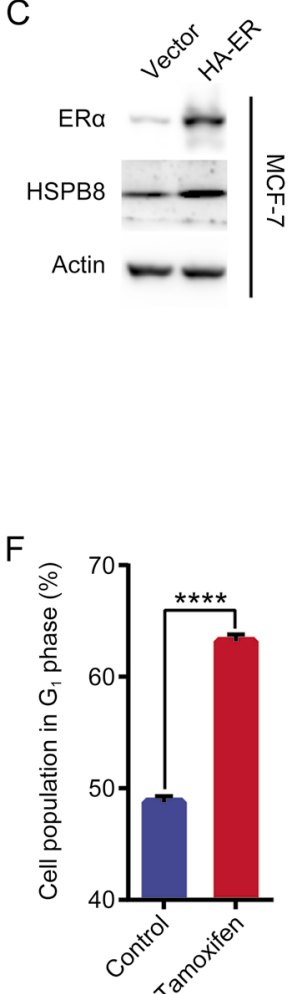

Figure 3. ER promotes HSPB8 expression. MCF-7 cells were treated with $100 \mathrm{nmol} / \mathrm{L} 17 \beta$-estradiol for $48 \mathrm{~h}$. The HSPB8 protein level was detected by Western blotting $(A)$, and the cell cycle distribution was assessed by flow cytometry (B). MCF-7 cells were transiently transfected with plasmid expressing HA-ER and cells were harvested for Western blotting (C) or cell cycle analysis (D) $48 \mathrm{~h}$ later. MCF-7 cells were treated with $1 \mu \mathrm{mol} / \mathrm{L}$ tamoxifen for $48 \mathrm{~h}$, and HSPB8 (E) and cell cycle distribution were detected (F).

$\mathrm{Rb}$ at Ser $807 / 811$ (Figure $4 \mathrm{~B}$ ), which play pivotal roles in the progression of the cell cycle from the $G_{1}$ to $S$ phase. Taken together, these results revealed that knocking down HSPB8 accumulated cells in the $G_{1}$ phase, which was accompanied by decreases in cyclin D1 and phosphorylated p-Rb.

To further confirm the effect of HSPB8 on cell cycle progression, MCF-7 cells were transiently transfected with plasmids expressing HSPB8 and the cell cycle distribution was analyzed. As shown in Figure 4C, forced expression of HSPB8 in MCF-7 cells rendered decrease in cell population in $G_{1}$ phase compared to cells transfected with empty vector. These results indicated that HSPB8 might play an important role in cell cycle progression.

\section{AZD8055 overcomes tamoxifen resistance by down-regulating the expression of HSPB8}

As we have observed over-expression of HSPB8 in tamoxifenresistant cells and that HSPB8 promoted cell cycle progression in breast cancer cells, we next searched for compounds with favorable activity against breast cancer cells over-expressing HSPB8. We used the online Cellminer database (https:// discover.nci.nih.gov/cellminer/) to search for potential compounds with anti-proliferative activity correlating with the expression of HSPB8. As shown in Figure 5A, the anti-proliferative activity of mTOR inhibitors, including X-387, Tem- sirolimus and rapamycin, and the PI3K/mTOR dual inhibitor GDC0980 were positively correlated with the expression of HSPB8 in NCI-60 screening. Of these, the mTOR kinase inhibitor $\mathrm{X}-387^{[20]}$ had the highest correlation coefficient $(0.50)$, followed by GDC0980, with a correlation coefficient of 0.43 . In contrast, the activity of rapamycin and its analog Temsirolimus was less correlated with HSPB8 expression, with correlation coefficients of 0.33 and 0.25 , respectively (Figure 5A). These results indicated that anti-proliferative activity derived from complete inhibition of mTOR might more strongly correlate with HSPB8 expression.

AZD8055, a first-in-class selective mTOR kinase inhibitor, was chosen for the following study ${ }^{[17]}$. We detected the antiproliferative activity of AZD8055 in MCF-7 and MCF-7/R cells. As shown in Figure $5 \mathrm{~B}$, the $\mathrm{IC}_{50}$ obtained with MCF-7/R cells was much lower than that with parent cells, indicating that MCF-7/R cells were more sensitive to AZD8055 than parent cells. We next detected the protein level of HSPB8 after AZD8055 treatment for 48 h. AZD8055 inhibited HSPB8 expression accompanied with decreased phosphorylation of 70S6K1 at Thr389 and Akt at Ser473 (Figure 5C). As AZD8055 induced $\mathrm{G}_{1}$ arrest in MCF-7 parent cells, we detected whether AZD8055 had the same effect in MCF-7/R cells. We found that AZD8055 treatment resulted in more than 80\% of MCF-7/ $R$ cells arresting in $G_{1}$ phase (Figure $5 D$ ). Combinations of 

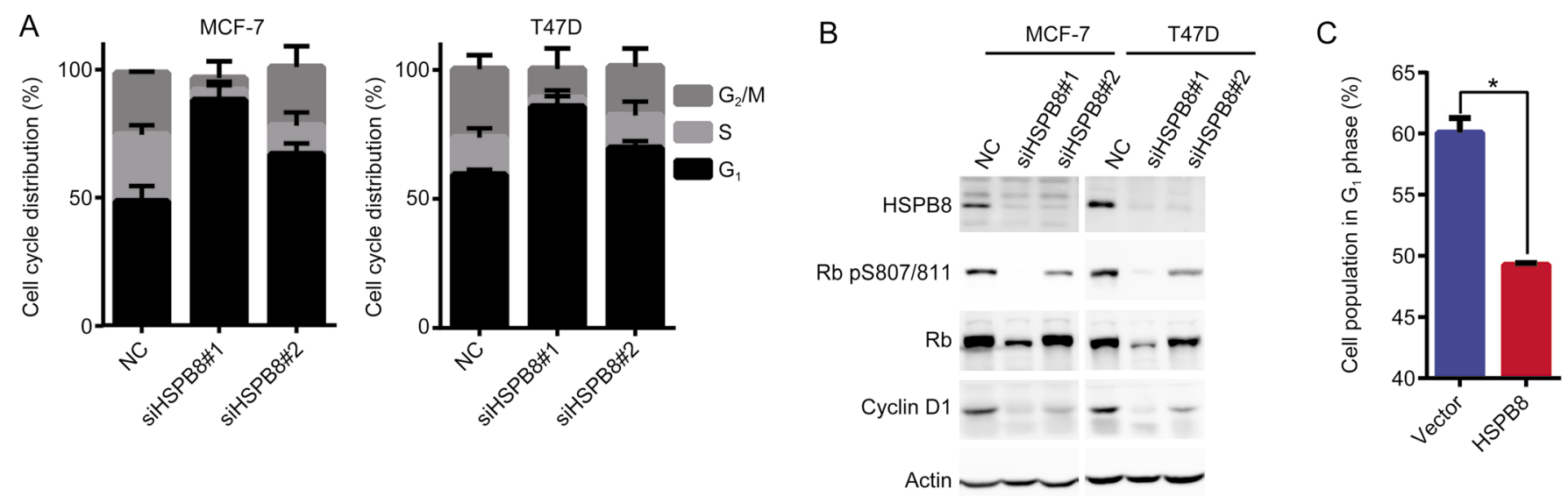

Figure 4. Knocking down HSPB8 induces cell cycle arrest. (A, B) MCF-7 and T47D cells were transfected with siRNAs targeting HSPB8 for $48 \mathrm{~h}$. Cell cycle distribution was detected by flow cytometry (A), and the indicated proteins were detected by Western blotting (B). (C) MCF-7 cells were transiently transfected with HSPB8-overexpressing plasmid for $48 \mathrm{~h}$, and the cell cycle distribution was analyzed.

AZD8055 and tamoxifen displayed an addictive effect against cell growth compared to tamoxifen treatment alone in MCF-7/ $\mathrm{R}$ cells, indicating that AZD8055 and tamoxifen may exert their activity in parallel pathways (Supplementary Figure S1). We also detected the activity of tamoxifen in MCF-7/R cells, where HSPB8 was down-regulated by a specific siRNA (Supplementary Figure S2). The siRNA and tamoxifen also had an addictive effect against cell growth compared to tamoxifen treatment in cells transfected with mock siRNA, suggesting that down-regulation of HSPB8 and tamoxifen acted independently against cell growth.

HSPB8 expression level positively correlates with the antiproliferative activity of AZD8055 in $\mathrm{ER}^{+}$cells

As HSPB8 expression is positively correlated with the antiproliferative activity of mTOR inhibitors in NCI60 screening, we investigated the correlation of HSPB8 expression and AZD8055 activity in a panel of breast cancer cells.

\begin{tabular}{cccc}
\hline A Compound & NSC number & Correlation coefficient & $P$ value \\
\hline X-387 & 756053 & 0.50 & $<0.001$ \\
GDC0980 & 764091 & 0.43 & 0.001 \\
Rapamycin & 758664 & 0.33 & 0.011 \\
Temsirolimus & 683864 & 0.25 & 0.062 \\
\hline
\end{tabular}
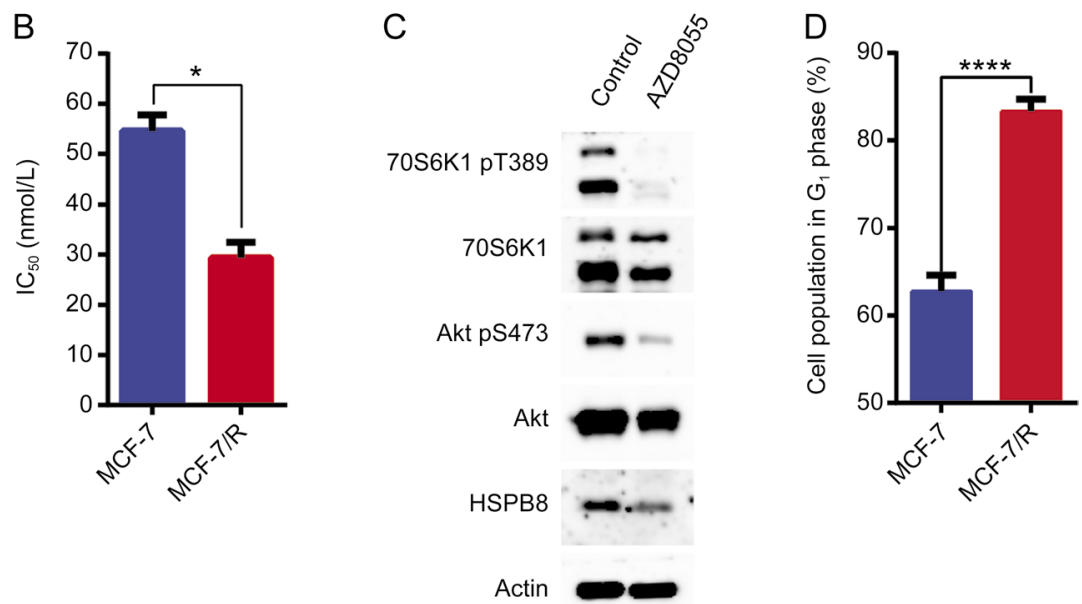

Figure 5. AZD8055 overcomes tamoxifen resistance by down-regulating the expression of HSPB8. (A) The correlation of HSPB8 expression with the anti-proliferative activity of X-387, GDC0980, Temsirolimus and rapamycin were analyzed using the Cellminer database. (B) Cells were treated with AZD8055 for $72 \mathrm{~h}$, and cell proliferation was assessed by the SRB assay. MCF-7/R cells were treated with $100 \mathrm{nM}$ AZD8055 for $48 \mathrm{~h}$, and HSPB8 (C) and cell cycle distribution were analyzed (D). 
A

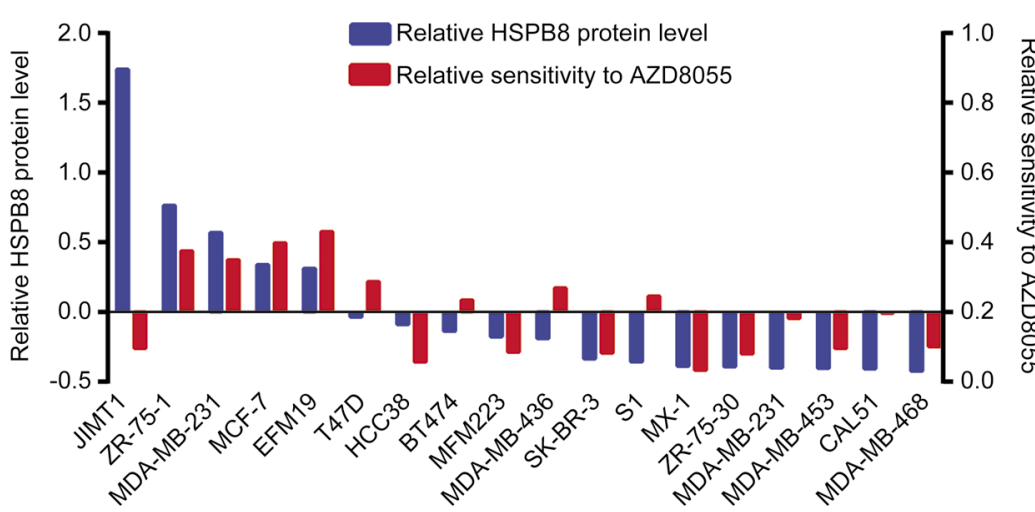

B

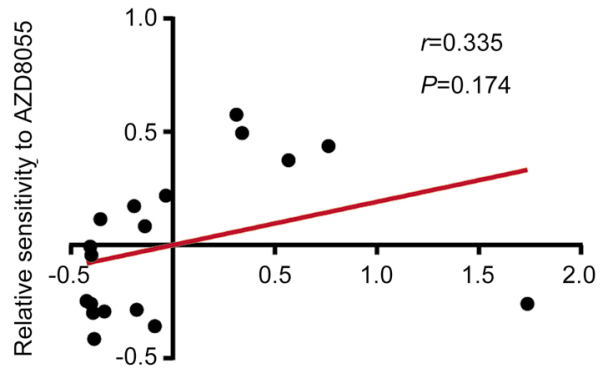

Relative HSPB8 protein level

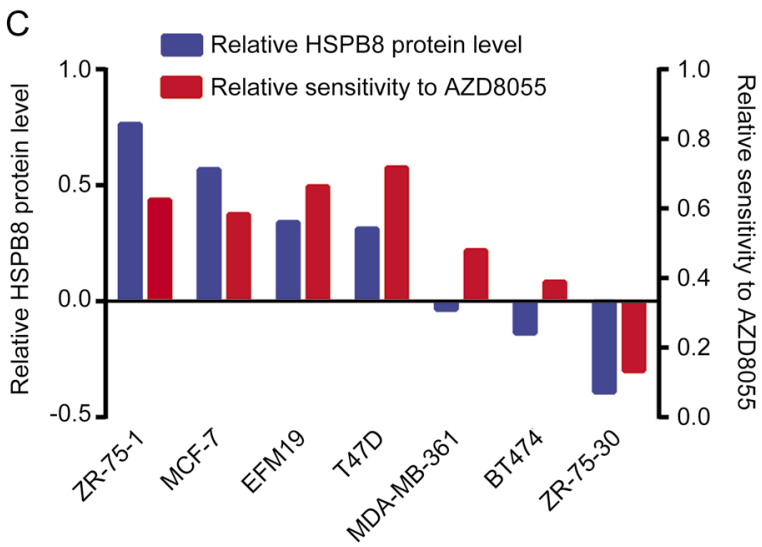

D

Figure 6. HSPB8 expression level positively correlates with the anti-proliferative activity of AZD8055 in ER ${ }^{+}$cells. (A) Relative HSPB8 protein level in 18 breast cancer cell lines was detected by Western blotting, and the relative anti-proliferative activity of AZD8055 was detected by SRB assay after treatment for $72 \mathrm{~h}$. (B) Correlation coefficients were calculated from the data presented in (A). (C, D) Correlation analysis of the HSPB8 expression level and the anti-proliferative activity of AZD8055 in $\mathrm{ER}^{+}$breast cancer cell lines was performed as in (A) and (B).

The relative protein level of HSPB8 and the anti-proliferative activity of AZD8055 represented as $\mathrm{GI}_{50}$ were detected in 18 breast cancer cell lines (Figure 6A). The correlation of AZD8055 activity and HSPB8 expression was plotted, and the correlation coefficient was calculated. As shown in Figure 6B, a correlation coefficient of 0.335 was obtained, which lacked statistical significance. Since mTOR inhibitors have been approved for the treatment of $\mathrm{ER}^{+}$breast cancer, the molecular classification of breast cancer was taken into consideration. We re-plotted the correlation of AZD8055 activity and HSPB8 expression in $\mathrm{ER}^{+}$cells (Figure $6 \mathrm{C}$ and $6 \mathrm{D}$ ). In contrast to the whole panel of tested breast cancer cell lines, the HSPB8 expression level significantly positively correlated with the anti-proliferative activity of AZD8055 in the sub-panel of $\mathrm{ER}^{+}$ cell lines, with a correlation coefficient of 0.852 . This positive correlation may reflect the regulation of HSPB8 by ER, which deserves further investigation.

\section{Discussion}

Breast cancer is the second leading cause of death among women $^{[1]}$. Endocrine therapy has achieved great success in $\mathrm{ER}^{+}$cancer patients ${ }^{[3]}$. However, de novo or acquired resistance prevents patients from benefitting from endocrine treat- ment. In this study, we report that HSPB8 is overexpressed in tamoxifen-resistant cells and that expression of HSPB8 correlates with poor prognosis in $\mathrm{ER}^{+}$patients. Down-regulation of HPSB8 induces $\mathrm{G}_{1}$ phase arrest in both parent and tamoxifen-resistant breast cancer cells. AZD8055 was found to be more potent against the proliferation of MCF-7/R cells than against that of parent cells, which was associated with downregulation of HSPB8. Finally, the anti-proliferative activity of AZD8055 is positively correlated with the protein expression level of HSPB8 in a panel of $\mathrm{ER}^{+}$breast cancer cells.

HSPB8 is a small heat shock protein that is relevant to tumor size, depth invasion, and lymph node metastasis ${ }^{[9,21]}$. We found that HSPB8 was overexpressed in tamoxifen-resistant MCF-7 cells, which is consistent with previous studies ${ }^{[9,13]}$. It has been reported that HSPB8 protected resistant cells from tamoxifen by blocking autophagy ${ }^{[9]}$. LMTK3 was reported as upstream of HSPB8 and increased the levels of HSPB8, thereby protecting MCF-7 cells from tamoxifen-induced cell death ${ }^{[13]}$. However, the role of HSPB8 in cell cycle progression is unknown. We found that down-regulation of HSPB8 in MCF-7/R cells resulted in enhanced cell population at $G_{1}$ phase, which is consistent with the decrease in the phosphorylation of $\mathrm{Rb}$ following the same treatment. Similarly, siRNAs 
against HSPB8 arrested MCF-7 and T47D cells in $\mathrm{G}_{1}$ phase, further supporting the notion that HSPB8 may also play a role in cell cycle progression. We also found that activation of ER induced HSPB8 expression. Interestingly, higher expression of HSPB8 was observed in $\mathrm{ER}^{+}$breast cancer patients than in ER patients, which may reflect the regulation of HSPB8 by ER. Gonzalez-Malerva et al reported that a high expression level of HSPB8 predicted an earlier relapse after tamoxifen treatment in a cohort of $277 \mathrm{ER}^{+}$breast cancer patients ${ }^{[9]}$. Similarly, we found that HSPB8 up-regulation correlated with poor survival in $\mathrm{ER}^{+}$patients but not $\mathrm{ER}^{-}$patients by analyzing data deposited in the cBioportal for Cancer Genomics database. Taken together, our results and previous studies indicated an important role of HSPB8 in resistance to tamoxifen, and its expression may predict poor prognosis after endocrine therapy. The mechanism of HSPB8 to mediate tamoxifen resistance may be associated with its role in cell cycle progression, autophagy and cell death, which deserve further investigation.

In an effort to discover a strategy to overcome the resistance of breast cancer to tamoxifen, we performed a comparative analysis using the Cellminer database ${ }^{[22]}$. mTOR inhibitors emerged as compounds whose activities were most significantly correlated with the expression of HSPB8 in NCI60 cell lines. Accordingly, AZD8055, an mTOR kinase inhibitor in clinical trials for the treatment of breast cancer, was more potent against tamoxifen-resistant cells than against parental cells. Consistent with our results, AZD8055 has been reported to be active against tamoxifen-resistant breast cancer cells ${ }^{[18]}$. We further demonstrated that AZD8055 was able to downregulate HSPB8 expression, which might be associated with its superior activity against tamoxifen-resistant cells with enhanced expression of HSPB8. Although we failed to test the activity of AZD8055 against MCF-7/R cells in vivo due to difficulty in establishing the xenograft model, mTOR inhibitors have been reported to be active against tamoxifen-resistant breast cancers in PDX-beard mice and in patients. Behrens et al demonstrated that everolimus combined with tamoxifen remarkably inhibited tumor growth (T/C 18\%), which was significantly superior to tamoxifen treatment alone $(\mathrm{T} / \mathrm{C}$ $48 \%$ ) in a tamoxifen-resistant PDX model ${ }^{[23]}$. Moreover, a randomized phase II trial of everolimus in combination with tamoxifen in metastatic breast cancer with prior exposure to aromatase inhibitors revealed that the 6-month clinical benefit rate was $61 \%$ (47 to 74 ) with tamoxifen plus everolimus and $42 \%$ (29 to 56) with tamoxifen alone. The time to progression (TTP) increased from 4.5 months with tamoxifen alone to 8.6 months with tamoxifen plus everolimus ${ }^{[24]}$. Furthermore, we revealed a significant correlation between HSPB8 expression and AZD8055 activity in a panel of $\mathrm{ER}^{+}$breast cancer cells. However, this correlation failed to be found in a cohort of mixed $\mathrm{ER}^{+}$and $\mathrm{ER}^{-}$breast cancer cell lines. This observation may further support the regulation of HSPB8 by ER signaling.

AZD8055 has been tested clinically for the treatment of advanced solid tumors. Available results showed that AZD8055 possessed an acceptable toxicity profile, but no RECIST responses were seen ${ }^{[25]}$. One of the reasons for clini- cal failure is that patients were recruited and treated without stratification based on biomarkers predicting the efficacy. For example, phosphorylated 4E-BP1 was suggested to predict the response of mTOR inhibitors ${ }^{[26,27]}$. The correlation of HSPB8 and AZD8055 activity in $\mathrm{ER}^{+}$breast cancer cells suggests that HSPB8 might be a potential predictive biomarker in the therapy of $\mathrm{ER}^{+}$patients with AZD8055, which deserves further validation in a larger cohort of in vitro and in vivo studies.

In summary, the present study demonstrates that AZD8055 could overcome tamoxifen resistance in breast cancers cells via inhibiting HSPB8 expression and the HSPB8 expression level might be a candidate predictive biomarker for the efficacy of AZD8055 in $\mathrm{ER}^{+}$breast cancers.

\section{Acknowledgements}

We thank Associate Professor Xiang WANG and Mr Yu-xiang WANG for their help with the cell culture and suggestions for the experimental design. This work was supported by the National Natural Science Foundation of China (81373445) and "Personalized Medicines-Molecular Signature-based Drug Discovery and Development", Strategic Priority Research Program of the Chinese Academy of Sciences (XDA12020202).

\section{Author contribution}

Jia-jie SHI, Si-meng CHEN, Chen-liang GUO and Ling-hua MENG designed the research; Jia-jie SHI, Si-meng CHEN, and Chen-liang GUO performed the experiments and analyzed the data; Yi-xue LI performed the bioinformatic analysis; Jiajie SHI and Ling-hua MENG drafted the manuscript; and Jian DING and Ling-hua MENG supervised the study.

\section{Supplementary information}

Supplementary information is available on the website of Acta Pharmacologica Sinica.

\section{References}

1 Siegel RL, Miller KD, Jemal A. Cancer Statistics, 2017. CA Cancer J Clin 2017; 67: 7-30.

2 Lobbezoo DJ, van Kampen RJ, Voogd AC, Dercksen MW, van den Berkmortel F, Smilde TJ, et al. Prognosis of metastatic breast cancer subtypes: the hormone receptor/HER2-positive subtype is associated with the most favorable outcome. Breast Cancer Res Treat 2013; 141: 507-14.

3 Lukong KE. Understanding breast cancer - The long and winding road. BBA Clin 2017; 7: 64-77.

4 Clarke R, Tyson JJ, Dixon JM. Endocrine resistance in breast cancer-An overview and update. Mol Cell Endocrinol 2015; 418: 220-34.

5 Milani A, Geuna E, Mittica G, Valabrega G. Overcoming endocrine resistance in metastatic breast cancer: Current evidence and future directions. World J Clin Oncol 2014; 5: 990-1001.

6 Chang M. Tamoxifen resistance in breast cancer. Biomol Ther (Seoul) 2012; 20: 256-67.

7 Ring A, Dowsett M. Mechanisms of tamoxifen resistance. Endocr Relat Cancer 2004; 11: 643-58.

8 Osborne CK, Schiff R. Mechanisms of endocrine resistance in breast cancer. Annu Rev Med 2011; 62: 233-47.

9 Gonzalez-Malerva L, Park J, Zou L, Hu Y, Moradpour Z, Pearlberg $J$, et al. High-throughput ectopic expression screen for tamoxifen 
resistance identifies an atypical kinase that blocks autophagy. Proc Natl Acad Sci U S A 2011; 108: 2058-63.

10 Chowdary TK, Raman B, Ramakrishna T, Rao CM. Mammalian Hsp22 is a heat-inducible small heat-shock protein with chaperone-like activity. Biochem J 2004; 381: 379-87.

11 Shemetov AA, Seit-Nebi AS, Gusev NB. Phosphorylation of human small heat shock protein HspB8 (Hsp22) by ERK1 protein kinase. Mol Cell Biochem 2011; 355: 47-55.

12 Carra S, Brunsting JF, Lambert H, Landry J, Kampinga HH. HspB8 participates in protein quality control by a non-chaperone-like mechanism that requires elF2 $\alpha$ phosphorylation. J Biol Chem 2009; 284: 5523-32.

13 Stebbing J, Filipovic A, Lit LC, Blighe K, Grothey A, Xu Y, et al. LMTK3 is implicated in endocrine resistance via multiple signaling pathways. Oncogene 2013; 32: 3371-80.

$14 \mathrm{MaXM}$, Blenis J. Molecular mechanisms of mTOR-mediated translational control. Nat Rev Mol Cell Biol 2009; 10: 307-18.

15 Shaw RJ, Cantley LC. Ras, $\mathrm{Pl}_{3} \mathrm{~K}$ and mTOR signalling controls tumour cell growth. Nature 2006; 441: 424-30.

16 Meng LH, Zheng XF. Toward rapamycin analog (rapalog)-based precision cancer therapy. Acta Pharmacol Sin 2015; 36: 1163-9.

17 Chresta CM, Davies BR, Hickson I, Harding T, Cosulich S, Critchlow SE, et al. AZD8055 is a potent, selective, and orally bioavailable ATPcompetitive mammalian target of rapamycin kinase inhibitor with in vitro and in vivo antitumor activity. Cancer Res 2010; 70: 288-98.

18 Jordan NJ, Dutkowski CM, Barrow D, Mottram HJ, Hutcheson IR, Nicholson Rl, et al. Impact of dual mTORC1/2 mTOR kinase inhibitor AZD8055 on acquired endocrine resistance in breast cancer in vitro. Breast Cancer Res 2014; 16: R12.

19 Li T, Wang J, Wang X, Yang N, Chen SM, Tong L, et al. WJD008, a dual phosphatidylinositol 3-kinase $(\mathrm{P} \mid 3 \mathrm{~K}) /$ mammalian target of rapamycin inhibitor, prevents $\mathrm{PI} 3 \mathrm{~K}$ signaling and inhibits the proliferation of transformed cells with oncogenic PI3K mutant. J Pharmacol Exp Ther 2010; 334: 830-8.

20 Chen SM, Liu JL, Wang X, Liang C, Ding J, Meng LH. Inhibition of tumor cell growth, proliferation and migration by $X-387$, a novel activesite inhibitor of mTOR. Biochem Pharmacol 2012; 83: 1183-94.

$21 \mathrm{Li} \mathrm{XS}, \mathrm{Xu}$ Q, Fu XY, Luo WS. Heat shock protein 22 overexpression is associated with the progression and prognosis in gastric cancer. J Cancer Res Clin Oncol 2014; 140: 1305-13.

22 Shankavaram UT, Varma S, Kane D, Sunshine M, Chary KK, Reinhold WC, et al. CellMiner: a relational database and query tool for the $\mathrm{NCl}-$ 60 cancer cell lines. BMC Genomics 2009; 10: 277.

23 Behrens D, Lykkesfeldt AE, Fichtner I. The mTOR pathway inhibitor RAD001 (everolimus) is highly efficacious in tamoxifen-sensitive and -resistant breast cancer xenografts. Target Oncol 2007; 2: 135-44.

24 Bachelot T, Bourgier C, Cropet C, Ray-Coquard I, Ferrero JM, Freyer $\mathrm{G}$, et al. Randomized phase II trial of everolimus in combination with tamoxifen in patients with hormone receptor-positive, human epidermal growth factor receptor 2-negative metastatic breast cancer with prior exposure to aromatase inhibitors: a GINECO study. J Clin Oncol 2012; 30: 2718-24.

25 Naing A, Aghajanian C, Raymond E, Olmos D, Schwartz G, Oelmann E, et al. Safety, tolerability, pharmacokinetics and pharmacodynamics of AZD8055 in advanced solid tumours and lymphoma. Br J Cancer 2012; 107: 1093-9.

26 Hong B, Wang H, Deng K, Wang W, Dai H, Lui VWY, et al. Combination treatment of RAD001 and BEZ235 exhibits synergistic antitumor activity via down-regulation of p-4E-BP1/Mcl-1 in small cell lung cancer. Oncotarget 2017; 8: 106486-98.

27 Fan QW, Nicolaides TP, Weiss WA. Inhibiting 4EBP1 in glioblastoma. Clin Cancer Res 2018; 24: 14-21. 